\title{
Exercise impacts the epigenome of cancer
}

\author{
De-Chen Lin ${ }^{1}$
}

Received: 4 May 2021 / Revised: 6 May 2021 / Accepted: 12 May 2021 / Published online: 27 May 2021

(c) The Author(s), under exclusive licence to Springer Nature Limited 2021

Physical excise improves health and decreases risk for many diseases, including cancer [1]. Epidemiological and observational studies [2] have shown that greater physical activity is associated with a reduced risk of multiple common cancer types, including those of the breast, prostate, colon and esophagus. It is imperative to understand the mechanisms underlying the contribution of physical excise to cancer pathophysiology since such knowledge will provide evidence for designing physical excise interventions in clinical trial settings. Moreover, mechanistic actions learned may also lead to new discoveries in cancer biology, which can help the development of innovative modalities for cancer prevention, early intervention and treatment.

Toward this end, there have been many studies aiming to understand the roles of physical excise in reducing cancer risk and improving outcome. Several mechanisms have been proposed [3], including (i) reducing sex hormone levels (e.g., estrogen and androgen); (ii) decreasing systemic inflammation; (iii) improving insulin resistance and obesity, which are risk factors for multiple cancer types; (iv) enhancing the function of immune cells. Clearly, these previous investigations have focused largely on environmental, extrinsic factors, which is not surprising given that physical excise indeed impacts the body as a whole. Conceivably, excise can also regulate tumor-intrinsic variables, however, such mechanistic studies have been few.

In the current issue of Prostate Cancer and Prostatic Diseases, Dufresne et al. [4] investigated specifically this question-whether and how physical excise regulates the cancer cell in a non-autonomous manner. Using a rat model implanted with a prostate cancer cell line (AT1), the authors compared the epigenome of cancer cells from sedentary control (CTL) with exercise training (ET) rats. Specifically,

De-Chen Lin

De-Chen.Lin@cshs.org

1 Cedars-Sinai Cancer, Department of Medicine, Cedars-Sinai Medical Center, Los Angeles, CA, USA they measured microRNA expression, DNA methylation and histone modifications. Interestingly, 75 microRNAs were differently expressed between the CTL and ET groups, and one of them was miR-27a-5p, which has been implicated in prostate cancer proliferation [5]. Exercise was also shown to increase global DNA methylation and downregulate the expression Dnmt genes in prostate cancer cells. However, the global level of histone H3 lysine acetylation was not changed in trained rats than sedentary rats. Coupled with their earlier finding [6] that trained rats had a slower prostate tumor growth with increased cancer cell apoptosis, these results indicate that physical exercise may benefit cancer control by altering the epigenetic regulation in prostate tumors.

In future investigations, it is warranted to pinpoint precise changes of the methylome and chromatin activity by deep sequencing-based methodologies, such as wholegenome bisulfite sequencing, chromatin Immunoprecipitation Sequencing, and Assay for Transposase-Accessible Chromatin using sequencing. These approaches will reveal which specific chromatin regions are regulated by physical excise, the magnitude of changes and the associated target genes.

Nevertheless, albeit largely observational, these findings are interesting as they add another layer of mechanism underlying physical excise in reducing cancer risk. Moreover, the involvement of epigenome in linking physical excise and cancer opens a new window for intervention and treatment, given that the cancer epigenome contains many promising drug targets [7]. Therefore, further understanding of the precise impact of physical excise on the cancer epigenome will promote the development and design of novel strategies to reduce cancer risk, early intervention and cancer treatment.

\section{Compliance with ethical standards}

Conflict of interest The author declares no competing interests. 
Publisher's note Springer Nature remains neutral with regard to jurisdictional claims in published maps and institutional affiliations.

\section{References}

1. Thune I, Furberg AS. Physical activity and cancer risk: doseresponse and cancer, all sites and site-specific. Med Sci Sports Exerc. 2001;33:S530-50.

2. Friedenreich CM, Orenstein MR. Physical activity and cancer prevention: etiologic evidence and biological mechanisms. J Nutr. 2002;132:3456S-64S.

3. McTiernan A. Mechanisms linking physical activity with cancer. Nat Rev Cancer. 2008;8:205-11.
4. Dufresne SGJ, Wong CP, Isanejad A, Ho E, Serna E, Gomez-Cabrera $\mathrm{M}$, et al. Exercise training as a modulator of epigenetic events in prostate tumors. Prostate Cancer Prostatic Dis. 2021. In press.

5. Barros-Silva D, Costa-Pinheiro P, Duarte H, Sousa EJ, Evangelista AF, Graca I, et al. MicroRNA-27a-5p regulation by promoter methylation and MYC signaling in prostate carcinogenesis. Cell Death Dis. 2018;9:167.

6. Gueritat J, Lefeuvre-Orfila L, Vincent S, Cretual A, Ravanat JL, Gratas-Delamarche A, et al. Exercise training combined with antioxidant supplementation prevents the antiproliferative activity of their single treatment in prostate cancer through inhibition of redox adaptation. Free Radic Biol Med. 2014;77:95-105.

7. Mohammad HP, Barbash O, Creasy CL. Targeting epigenetic modifications in cancer therapy: erasing the roadmap to cancer. Nat Med. 2019;25:403-18. 